\title{
Norois
}

Environnement, aménagement, société

\section{Contributions géographiques à la définition d'une culture du risque en milieu littoral : le cas des résidents de l'île d'oléron (Charente-Maritime, France)}

Geographic contributions to the definition of a risk culture in coastal areas: the case of residents of the island of Oléron (Charente-Maritime, France)

\section{David Chionne}

\section{(2) OpenEdition}

Journals

\section{Édition électronique}

URL : https://journals.openedition.org/norois/7343

DOI : $10.4000 /$ norois. 7343

ISBN : 978-2-7535-7873-9

ISSN : 1760-8546

Éditeur

Presses universitaires de Rennes

Édition imprimée

Date de publication : 19 juin 2019

Pagination : 27-48

ISBN : 978-2-7535-7871-5

ISSN : 0029-182X

Référence électronique

David Chionne, "Contributions géographiques à la définition d'une culture du risque en milieu littoral : le cas des résidents de l'île d'Oléron (Charente-Maritime, France) », Norois [En ligne], 250 | 2019, mis en ligne le 01 janvier 2022, consulté le 16 février 2023. URL : http://journals.openedition.org/norois/7343 ; DOI : https://doi.org/10.4000/norois.7343

\section{cc) (†)}

Creative Commons - Attribution - Pas d'Utilisation Commerciale - Pas de Modification 4.0 International - CC BY-NC-ND 4.0

https://creativecommons.org/licenses/by-nc-nd/4.0/ 


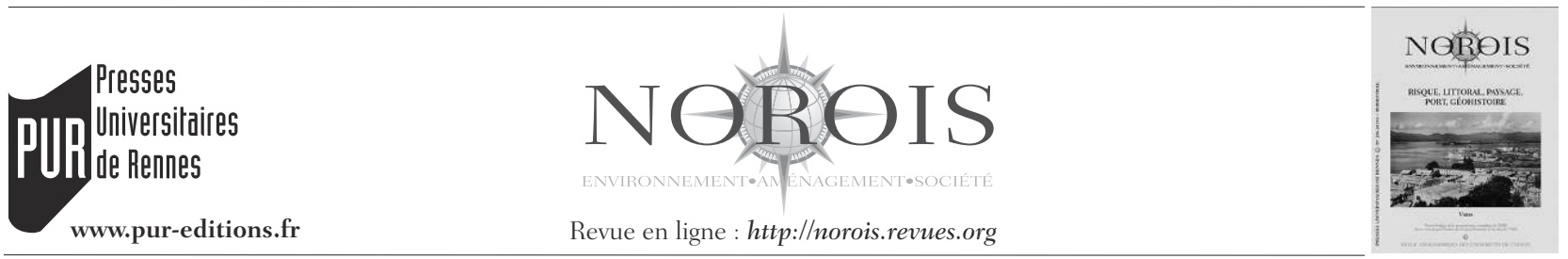

\title{
Contributions géographiques à la définition d'une culture du risque en milieu littoral : le cas des résidents de l'île d'Oléron (Charente-Maritime, France)
}

\author{
Geographic Contributions to the Definition of a Risk Culture in Coastal Areas: \\ The Case of Residents of the Island of Oléron (Charente-Maritime, France)
}

\author{
David Chionne
}

Laboratoire de Géographie Physique, CNRS, Environnements quaternaires et actuels, UMR 8591, - Batiment Y,

1, place Aristide-Briand, 92195 Meudon cedex, France (david.chionne@cnrs.fr)

\begin{abstract}
Résumé : Le point de départ de cette étude a été l'expression de «culture du risque »(CDR) telle qu'elle a été employée par l'État suite aux événements liés à la tempête Xynthia en 2010. À travers celle-ci, ce dernier insiste sur le fait que les populations se caractériseraient par un manque de connaissances et de comportements inadaptés vis-à-vis des aléas côtiers. Parallèlement, il affiche sa volonté de favoriser leur sensibilisation vis-à-vis de ces problématiques. Cependant, nous avons constaté qu'un dispositif législatif de communication et de sensibilisation existe depuis les années 1980, mais que son bilan paraît toujours mitigé. De plus, deux visions principales paraissent s'affronter : d'un côté, l'État qui cherche à limiter l'implantation des enjeux dans des zones dites «à risque », de l'autre les populations locales qui demandent à protéger leurs enjeux.

Ainsi, la confrontation aux mêmes aléas ne donne pas lieu à des interprétations identiques : différentes cultures du risque existent. Afin de comprendre ces différences, nous tenterons de démontrer l'hypothèse stipulant que les différentes attitudes observées auprès des individus en matière de risques côtiers sont déterminées par leurs relations au milieu. Plus précisément, il sera question de caractériser ces rapports et d'illustrer en quoi ceux-ci sont susceptibles d'affecter leurs discours à propos des aléas côtiers. Cela nous amènera à définir les composantes d'une culture du risque et d'en évaluer le niveau auprès des résidents oléronais. Pour cela, nous nous appuierons sur l'analyse statistique de résultats issus d'une enquête par questionnaire, mais aussi sur l'analyse de cartes mentales.
\end{abstract}

\begin{abstract}
The starting point of this study was the expression "risk culture" as used by the French government following the events related to the storm Xynthia in 2010. Through this, it emphasizes on the fact that populations are characterized by a lack of knowledge and inappropriate behaviours when faced with coastal hazards. At the same time, it demonstrates its desire to raise public awareness of these issues. However, we have noted that a legislative communication and awareness-raising framework has been in place since the 1980s, but its results are still limited. In addition, two main visions seem to be confronting each other: on the one hand, the State, which seeks to limit the establishment of stakes in so-called "at-risk" areas, and on the other hand, the local inhabitants, who ask to protect their stakes.

Thus, confronting the same hazards does not lead to identical interpretations: different cultures of risk exist. In order to understand these differences, we will attempt to demonstrate the hypothesis that the different attitudes observed among populations regarding coastal risks are determined by their relationship to the environment. More specifically, the aim is to characterize these relationships and illustrate how they are likely to affect their discourses about coastal hazards. This will lead us to define the components of a risk culture and assess its level among Oleronian residents. To do this, we will rely on the statistical analysis of results from a questionnaire survey, but also on mental maps.
\end{abstract}


Mots clés : Aléas côtiers - submersion marine - culture du risque - représentations - comportements - cartes mentales

Keywords: Coastal hazards, marine submersion, risk culture, representations, behaviours, mental maps

\section{INTRODUCTION}

L'île d'Oléron (Charente-Maritime), située sur la façade atlantique, est la $2^{\mathrm{e}}$ île de France métropolitaine, de par sa surface de $174 \mathrm{~km}^{2}$. Elle se caractérise par des côtes basses, sableuses et humides : les surfaces situées à moins de $250 \mathrm{~m}$ présentent une altitude moyenne de $5 \mathrm{~m} \mathrm{NGF}^{1}$. Régulièrement affectée par les phénomènes d'érosion et de submersion marines, elle a connu trois événements majeurs : en 1957, 1999 et 2010. Plus précisément, les aléas de submersion marine concernent plutôt la façade est, tandis que l'érosion la côte atlantique, à l'ouest (fig. 1 - planche III). $30 \%$ de l'île sont exposés à ces phénomènes, et plus particulièrement, $13 \%$ des zones urbaines (d'après le croisement des données issues du programme CORINE Land Cover de 2012). Malgré une circulaire d'août 2011 incitant à un déploiement rapide de Plans de Prévention des Risques Naturels (PPRN) spécifiquement « littoraux » pour la totalité des communes de l'île dont la forte vulnérabilité a été révélée lors de la tempête Xynthia de 2010, ces documents de servitude d'utilité publique sont toujours en révision.

La conjonction de la tempête Xynthia et de fortes marées, en février 2010, ont rappelé la présence de ces aléas. En effet, une partie de son territoire a été submergée et des reculs de 10 à 20 mètres ont été enregistrés en de nombreux points de la façade atlantique (Duvat, 2010). Cette situation a conduit les collectivités, à réfléchir sur la vulnérabilité de cet espace face à ces phénomènes et à redéfinir des stratégies de prévention. Ainsi, nous avons été amenés à travailler avec la Communauté de Communes de l'île d'Oléron, afin de mieux cerner les relations que les habitants - en particulier les résidents secondaires - entretiennent avec les aléas côtiers (submersions marines et érosion côtière).

1. À titre indicatif, $48 \%$ des zones urbanisées à moins de $250 \mathrm{~m}$ de la côte ont une altitude inférieure à $5 \mathrm{~m}$ NGF.

\section{Absence de «culture du risque »}

Dans ce cadre, le point de départ de cette recherche a été l'expression « absence de culture du risque ». Celle-ci se retrouve dans les principaux rapports gouvernementaux, réalisés suite au bilan dramatique suite au passage de la tempête Xynthia. Le rapport sénatorial Anziani (2010) propose une définition plutôt succincte de ce qu'est une « culture du risque » : il s'agirait d'un « ensemble de connaissances et de pratiques qui permet aux individus d'agir efficacement vis-à-vis des aléas de la nature et d'en limiter les dégâts ». Selon l'État, ce «manque de culture du risque » rend difficile l'application de sa stratégie de prévention. Plus précisément, il souligne le manque de connaissances et les comportements inadaptés vis-à-vis des risques côtiers et affiche sa volonté de favoriser la sensibilisation des populations. Cela étant dit, ces directives concernant la sensibilisation existent depuis les années 1980. En effet, depuis la loi nº 87-565 du 22 juillet 1987, il est clairement établi que les citoyens ont droit à l'information sur les risques majeurs et les mesures de sauvegarde qui les concernent. Au fil des années, cette disposition sera complétée, notamment par la définition des compétences des acteurs de l'administration publique en matière de communication $^{2}$ et en obligeant ceux-ci à communiquer sur le sujet ${ }^{3}$. Malgré ce dispositif réglementaire, le bilan paraît mitigé. L’enquête réalisée par nos soins confirmera cette situation relevée par les différents rapports gouvernementaux.

Au-delà des questions de communication, nos premières enquêtes sur le terrain nous ont permis de mettre en évidence que la nature de cet antagonisme découlait de différentes conceptions des

2. Décret n. 90-918 du 11 octobre 1990 : partage des compétences pour l'élaboration et la diffusion des documents d'information (DDRM, DICRIM) entre le préfet et le maire.

3. Article L125-2 du Code de l'Environnement (2004) : dans les communes où un PPR est présent, le maire doit informer ses administrés au moins une fois tous les deux ans; article R125-23 du Code de l'Environnement (2005) : lors des transactions immobilières, devra être annexé aux contrats un « état des risques »; article R563-11 du Code de l'Environnement (2005) : le maire doit implanter des repères de crues indiquant le niveau atteint par les PHEC et les signaler. 
problèmes liés aux aléas côtiers et par conséquent des solutions envisagées. Deux visions principales paraissent en effet s'affronter. D'un côté, l'État qui cherche à limiter l'implantation des enjeux dans des zones dites «à risque ". De l'autre, les populations locales qui demandent à protéger leurs enjeux, voire des collectivités qui souhaitent préserver leur espace foncier. Cette opposition se traduirait par une mise en place difficile des Plans de Prévention des Risques qui définissent, à l'échelle infracommunale, l'usage des espaces en fonction de l'intensité des aléas susceptibles de s'y produire. Le Bourhis (2007) et Vinet (2007) constataient déjà que l'élaboration des PPR progresse de façon très modérée : les objectifs que l'État se fixe régulièrement en termes de nombre de plans approuvés ne sont jamais atteints. Cela est particulièrement le cas pour les territoires littoraux où nous avons pu effectivement évaluer, à partir de la base de données GASPAR (Gestion Assistée Procédures Administratives relatives à la gestion des Risques), qu'en 2013 seulement $17 \%$ des communes littorales soumises à un aléa littoral disposent d'un PPR approuvé. Cette situation est en grande partie à l'origine de l'aggravation du bilan lié à la tempête Xynthia dont les inondations se sont pourtant limitées à des espaces déjà submergés par le passé (Garnier et al., 2010) et qui auraient donc dû être régulés par les PPR. Comment pouvonsnous expliquer cet échec, alors qu'il s'agit d'une stratégie en œuvre depuis plus de trois décennies?

En ce qui concerne notre étude, l'illustration de cette opposition a été les manifestations qui ont suivi la décision de l'État de déconstruire une part importante du bâti inondé suite à la tempête Xynthia en 2010. Par exemple, dans les quartiers de Boyardville et de La Perrotine, suite aux submersions liées à la tempête, l'État a durci sa stratégie, consistant à réguler l'implantation des enjeux par rapport à l'intensité des aléas. Des «zones de danger extrême » ont été définies, dans lesquelles il était prévu de déconstruire les habitations. Face à cette démarche, les populations locales ont manifesté leur opposition, demandant plutôt de renforcer les protections de leurs enjeux. Cependant, il est important de noter que cet antagonisme, largement relayé par les médias, est plutôt caricatural. En effet, si les médias ont mis particulièrement en exergue ceux qui ont résisté aux politiques de déconstruction de l'État (De Vanssay et al., 2011), une part non négligeable des populations touchées par les submersions ou voisines de celle-ci a décidé de déménager. Ainsi, dans ces zones d'extrême danger, dites «zones de solidarité » (fig. 2 - planche IV), nous avons pu observer plusieurs stratégies : des résidents ont choisi de vendre leur parcelle (représentés par parcelles vertes), d'autres ont préféré rester (les parcelles rouges). Ces territoires se caractérisent alors par un mitage singulier : ce paysage ne résulte pas de l'extension du bâti, mais plutôt de son retrait.

Cela nous amène à considérer un postulat bien établi en sciences humaines : que le risque est une « construction sociale»(Peretti-Watel, 2000). C'està-dire que face à un même aléa, les évaluations du risque seront différentes selon les individus, ce qui aboutit à des comportements différents. L'intérêt de comprendre ces différentes attitudes nous paraît donc évident, dans la mesure où cela permettrait de faciliter le dialogue entre les différents acteurs concernés par les aléas côtiers : des décideurs publics aux résidents. Plusieurs éléments nous ont amenés à poser une première hypothèse stipulant que les différentes attitudes observées auprès des populations en matière de risques côtiers sont déterminées par leurs relations particulières au milieu.

\section{Renouvellement de la population oléronaise et des activités}

En effet, la diversité des manières de voir et d'agir face aux aléas côtiers et les difficultés rencontrées lors de la mise en place de la stratégie de régulation de l'État nous semblent en partie dues aux relations qu'entretiennent les populations locales avec les espaces littoraux. Ces individus sont plus sensibles aux aménités inhérentes à ces territoires qu’à leurs contraintes. Plusieurs auteurs ont expliqué cette attitude par : une urbanisation massive du littoral, en œuvre depuis le XIX ${ }^{\mathrm{e}}$ siècle et s'accélérant à partir de la deuxième moitié $\mathrm{du} \mathrm{xx}^{\mathrm{e}}$, amenant une population exogène qui remplace progressivement, en grande partie, celle qui est traditionnellement liée à ces milieux par ses activités (Morel et al., 2004); une relative accalmie des événements climatiques pendant cette même période (Pigeon, 2010); la confiance de l'Homme en sa capacité à maîtriser la Nature grâce au progrès technique, depuis l'avènement de l'industrialisation (Meschinet de Richemond et Reghezza, 2010). 
Ces tendances se sont accompagnées de deux phénomènes. L'un directement observable : une densification telle que les communes littorales métropolitaines présentent des valeurs 2,5 fois supérieures à la moyenne nationale, accueillant $10 \%$ de la population nationale sur seulement $4 \%$ du territoire (IFEN, 2007). De plus, dans l'attente d'une augmentation d'environ 3 millions d'habitants supplémentaires d'ici 30 ans (SOeS, 2011), les espaces deviennent de plus en plus convoités. L'autre conséquence difficilement mesurable de ce renouvellement s'identifie, selon nous, par une « urbanisation des mœurs » (Cartier, 2004). Elle s'est traduite par des choix, pris tant au niveau des collectivités locales que de leurs administrés, à travers lesquels les questions environnementales et celles liées aux aléas ont été souvent reléguées en arrière-plan, soit par méconnaissance soit délibérément. Ainsi, l'étalement urbain dans des zones déjà inondées par le passé qui est une des principales causes du lourd bilan suite à la tempête Xynthia, est à comprendre non pas comme un simple problème d'incivilité face à la législation, mais plutôt comme étant lié à une relation particulière que les populations entretiennent avec leur milieu. Les évaluations étatiques et locales des risques, reposant sur des critères différents, aboutissent à des stratégies divergentes.
L'île d'Oléron a connu une densification et un étalement des installations humaines très importants depuis la deuxième moitié $\mathrm{du} \mathrm{Xx}^{\mathrm{e}}$ siècle. En effet, on observe une forte augmentation de la population oléronaise depuis les années 1950. Si cette augmentation précède la mise en service du pont reliant l'île au continent en 1966, le pont et sa gratuité en 1991 semblent avoir davantage impacté l'implantation de résidences occasionnelles (I'INSEE comptabilise dans les résidences occasionnelles, à la fois, les résidences secondaires et les résidences de tourisme). Ainsi, $60 \%$ des logements oléronais sont occasionnels, alors que la moyenne des communes, du littoral métropolitain, est autour de $25 \%$. La forte augmentation des résidents est principalement due à un solde migratoire positif (représenté par les barres en vert), compensant un solde naturel négatif (en orange) : ce qui montre bien un renouvellement de la population. Par ailleurs, on observe une augmentation du secteur tertiaire aux dépens du secteur primaire. Ainsi, les activités traditionnelles, liées à l'aquaculture (notamment l'ostréiculture, la pèche) et aussi l'agriculture, sont remplacées essentiellement par des activités liées au tourisme et des services d'aide à la personne.

Par ailleurs, en termes culturels, à l'île d'Oléron, on remarquera des implantations discutables, comme

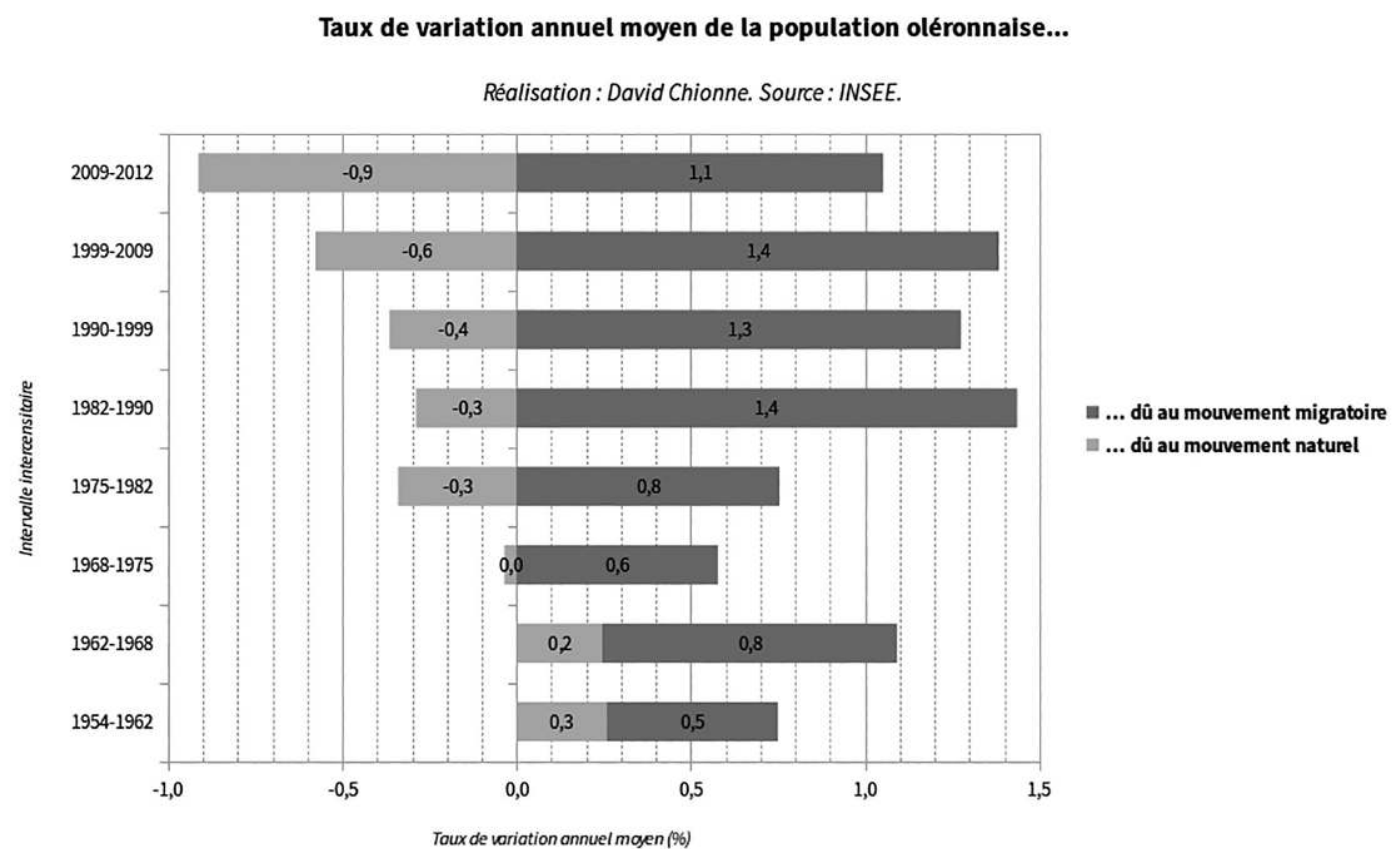

Figure 3 : Renouvellement de la population oléronaise Renewal of Oléron's population 
ces habitations récentes sur les dunes de la côte ouest; ou aussi la construction de lotissements dans des zones basses, exposées. Cependant, les historiens, tels que Péret et Sauzeau (2014), ont aussi mis en évidence un changement dans les connaissances et les comportements vis-à-vis des aléas côtiers. En effet, le quartier de la Perrotine a été construit sur des polders (appelées localement tannes), donc sur des terres gagnées sur la mer. Il était équipé d'un système de canaux de drainage, géré par les résidents, permettant d'évacuer les eaux en cas de submersion. Lors de la submersion de 1957, comparable à celle liée à Xynthia, les habitants du quartier ont su manœuvrer les vannes (appelées localement varaignes), limitant les dégâts de la mer. Alors qu'en 2010 plusieurs facteurs ont rendu ce système inopérant : en particulier, la perte de savoirs, le changement de propriétaire, souvent devenus secondaires (donc absents de leur résidence) et la privatisation des parcelles. Ces observations nous amènent donc à nous demander si ce renouvellement de la population oléronaise ne conduirait pas à un découplage entre les habitants et leur milieu.

\section{Problématique}

Ainsi, nous avons mis en évidence trois postulats de départ qui ont abouti à la définition de notre problématique. Tout d'abord, il existe différentes manières de voir et d'agir face aux aléas côtiers. Ensuite, nous avons observé qu'une partie de la population oléronaise a été progressivement renouvelée et que cette situation particulière est susceptible d'induire des connaissances et des comportements inadaptés, c'est-à-dire à un découplage entre les habitants et leur milieu. Ces postulats nous ont amenés à définir une problématique composée de deux questions principales : quels facteurs influencent une culture du risque donnée? Et, parmi ceux-ci, en quoi ce découplage habitantsmilieu influe-t-il sur la culture du risque?

Adoptant une approche quantitative, visant à analyser statistiquement les discours recueillis par le biais de questionnaires et de cartes mentales, une attention particulière a été portée à la définition des variables dont nous voulons vérifier la dépendance. Nous avons donc tenté en un premier temps de traduire le concept de «culture du risque », les caractéristiques des individus et de leur relation au milieu côtier en variables. Ensuite, nous avons testé les relations de dépendance entre ces différentes variables pour être en mesure d'illustrer en quoi ce découplage résidents-milieu est susceptible d'affecter les attitudes des résidents oléronais à propos des aléas côtiers et par conséquent leur culture du risque. Enfin, si ce travail porte sur les manières de voir et d'agir face aux aléas côtiers, il a aussi pour objectif d'engager des réflexions sur le sujet, plus vaste, des relations entre les sociétés et les aléas naturels.

\section{MÉTHOdE}

\section{Définition des outils d'enquête}

Afin de traduire le concept de «culture du risque », nous avons mobilisé la définition initiale, que l'on retrouve dans les principaux rapports gouvernementaux. Ainsi, selon le rapport Anziani (2010), la « culture du risque » se définit comme un « ensemble de connaissances et de pratiques qui permet aux individus d'agir efficacement vis-àvis des aléas de la nature et d'en limiter les dégâts ». $\mathrm{La}$ « culture du risque » peut se résumer en un ensemble de caractères décrivant les attitudes des individus vis-à-vis des aléas côtiers. Deux catégories principales peuvent se distinguer : les connaissances et les comportements. Ces deux éléments constituent le noyau dont on veut en évaluer le niveau auprès d'une population donnée. Par connaissance, nous entendons la capacité d'un individu à évaluer l'exposition de son logement à un aléa côtier donné, ainsi que sa connaissance des pratiques de mise en sécurité des biens et des personnes en cas de crise ou hors période de crise (prévention).

À partir de la synthèse d'enquêtes académiques portant sur les relations entre sociétés et environnement du littoral métropolitain ${ }^{4}$, mais aussi dans d'autres contextes ${ }^{5}$, nous avons construit un système composé de plusieurs facteurs susceptibles de faire varier ce noyau, que nous reportons dans le

\footnotetext{
4. Pour chaque enquête, nous reportons le nom du programme, l'année de conclusion et la région étudiée : PNEC (2004) Nord-Pas-de-Calais ; Université Paris 1 (2006) Languedoc-Roussillon; BEACHMED-e/InterregIIIC Sud (2007) Languedoc-Roussillon; MISSEEVA (2010) LanguedocRoussillon; Liteau/BARCASUB (2011) Aquitaine; GICC/ADAPTALIT (2011); Expertise Syndicat mixte Grand littoral picard (2011) Picardie; COCORISCO (2013) Bretagne; CRISSIS/CFSRS (2016) LanguedocRoussillon; Liteau/SOLTER (2015) Languedoc-Roussillon.

5. Autres enquêtes non centrées sur les risques côtiers ou s'étant déroulées hors du littoral métropolitain : RINAMED (2004), Defossez (2006), TSUNARISQUE (2006), Heitz (2005, 2009) et D'Ercole (1996).
} 
tableau ci-dessous (tabl. 1). Plusieurs thématiques sont abordées : les caractères démographiques classiques, les activités professionnelles et les loisirs (en tenant compte de la proximité de ceux-ci au milieu naturel), la fréquentation de l'île d'Oléron, la représentation de l'environnement (en particulier de la mer, mais aussi de l'île au quotidien) et enfin les sources d'information : c'est-à-dire par quels biais les individus ont-ils acquis leurs connaissances au sujet des aléas côtiers. Il peut s'agir, par exemple, d'informations issues de leur propre expérience ou qu'ils auraient recueillies à travers les médias.

Pour bien comprendre la manière dont les individus évaluent les risques et agissent en conséquence, nous avons étudié les mécanismes cognitifs, c'est-à-dire, comment les individus se saisissent de l'environnement qui les entoure, ainsi que leurs représentations. Notre position a été d'une part, de montrer que ces représentations sont à la fois issues d'expériences individuelles et collectives (notamment en s'appuyant sur les travaux de Joffe, 2003) et, d'autre part, que les représentations que l'on a du « réel » conditionnent les comportements vis-à-vis de celui-ci (selon, entre autres, Moscovici, 1961). En ce qui concerne la façon d'évaluer les discours recueillis, au sujet des risques, nous avons suivi deux approches majeures : psychométrique (Slovic, 2000), c'est-à-dire, où l'on évaluerait les discours obtenus par rapport à une référence donnée et culturaliste (Douglas et Widalvsky, 1982), qui chercherait à mettre en évidence le contexte socioculturel de production d'un discours donné. Ainsi, les discours des résidents oléronais ont été comparés aux informations officielles, ce qui permet d'analyser par exemple l'impact de la communication officielle. Mais en même temps, nous avons tenté de comprendre dans quelle mesure les rapports que les Oléronais entretiennent avec leur île influent sur leurs discours et leurs comportements.

Les différents caractères décrivant la culture du risque des individus et leur contexte ont été transposés dans un questionnaire de 36 questions. La formulation et l'enchaînement de ces questions ont été établis suite à des essais sur un échantillon de 30 résidents oléronais. Enfin, deux questions ont été posées sous la forme de deux cartes à remplir, des cartes mentales : l'une demandant de représenter

\begin{tabular}{|c|c|c|c|}
\hline TYPE DE VARIABLE & THÈME & DÉTAIL & QUESTION N $^{\circ}$ \\
\hline \multirow{7}{*}{$\begin{array}{l}\text { VARIABLES À EXPLIQUER : } \\
\text { Composantes de la culture du } \\
\text { risque }\end{array}$} & \multirow{2}{*}{ 1. Connaissance } & Des aléas & 5,6 \\
\hline & & Des moyens d'y faire face & 8,16 \\
\hline & \multirow{2}{*}{ 2. Évaluation du risque } & Propre & 9 \\
\hline & & De son environnement proche & Carte 1 \\
\hline & \multirow[t]{2}{*}{ 3. Comportements } & $\begin{array}{l}\text { Hors situation de crise (préven- } \\
\text { tion) }\end{array}$ & $7,8,25$ \\
\hline & & Pendant situation de crise & 11, 12, Carte 2 \\
\hline & 4. Opinions & Liées à la gestion des risques & $8,15,16$ \\
\hline \multirow{10}{*}{$\begin{array}{l}\text { VARIABLES EXPLICATIVES : } \\
\text { Caractères contextuels }\end{array}$} & \multirow{2}{*}{ 5. Sources d'information } & Directe (vécu, mémoire) & 6,13 \\
\hline & & Indirecte (médias) & $6,13,14$ \\
\hline & \multirow{4}{*}{ 6. Caractéristiques individuelles } & Sexe & 33 \\
\hline & & Âge & 34 \\
\hline & & Études & 35 \\
\hline & & Occupants logement (enfants) & 24 \\
\hline & \multirow{2}{*}{ 7. Pratiques de l'espace } & $\begin{array}{c}\text { Activités (professionnelles et } \\
\text { extra-p.) }\end{array}$ & $26,27,28,29,30,31,32$ \\
\hline & & $\begin{array}{l}\text { Fréquentation (durée, saisons, } \\
\text { lieux) }\end{array}$ & $17,18,19,20,21,22,23$ \\
\hline & 8. Représentations de l'espace & $\begin{array}{c}\text { Milieu et préoccupations quoti- } \\
\text { diennes }\end{array}$ & $1,2,3,4,10,36$ \\
\hline & 9. Localisation & $\begin{array}{c}\text { Par rapport à la mer et aux zones } \\
\text { sinistrées }\end{array}$ & Coordonnées du logement \\
\hline
\end{tabular}

Tableau 1 : Types de variables recueillies Types of collected variables 
les espaces potentiellement submersibles et l'autre demandant de tracer les chemins empruntés pour se réfugier en cas de submersion.

\section{Choix des sites d'enquête}

Dans la mesure où notre enquête a pour objet d'étudier les relations entre individus et aléas côtiers et, plus généralement, le milieu côtier, nous avons apporté une attention particulière au choix des sites d'enquête. L'île d'Oléron se caractérise par une mosaïque de paysages, issus de dynamiques et d'interactions complexes, entre société et nature. Afin de tenir compte de cette diversité, nous avons établi une typologie des différents types de côtes basses.

Deux catégories principales ont été déterminées (cf. fig. 1 - planche III) : les côtes dunaires qui se trouvent principalement sur la façade ouest de l'île, soumises principalement à des problèmes d'érosion, et les côtes à marais, tournées quasi exclusivement vers le continent, à l'est, concernées surtout par des submersions. Ensuite, nous avons tenu compte de 4 critères afin de choisir les sites : leur exposition aux aléas (selon les zonages des PPR), leur submersion réelle (lors de la tempête Xynthia) et leur distance à la mer. Ainsi, nous avons pu interroger des individus à la fois exposés ou non et sinistrés ou non. Enfin, les sites d'enquête ont été choisis selon la densité du bâti, afin que l'on puisse aisément atteindre des effectifs satisfaisant par site. L'échantillon ciblé est l'ensemble des résidents oléronais, quelle que soit leur durée de résidence (principaux, secondaires, touristes).

\section{RÉSULTATS ET INTERPRÉTATIONS}

Suite à nos enquêtes sur le terrain, nous avons obtenu les discours de 453 individus par questionnaire. Certaines réponses, issues de questions ouvertes, ont nécessité une phase de recodage assez longue afin de les rendre statistiquement exploitables. De plus, nous avons recueilli 2 cartes mentales pour 451 individus (902 au total). En ce qui concerne ces dernières, dans la mesure où les individus ont employé différents modes de représentation, il a fallu extraire leurs réponses manuellement et les compiler dans un SIG. Étant donné la durée de ces traitements, 570 cartes produites par 285 individus ont été analysées.
La population oléronaise de référence se caractérise par un âge moyen de plus de 60 ans. Nous retrouvons une valeur similaire dans notre échantillon, bien que les classes d'âges les plus élevées (plus de 60 ans) soient surreprésentées. Les catégories les plus âgées sont sans doute les moins mobiles, donc plus facilement accessibles. Notre échantillon se distingue par une surreprésentation des diplômes les plus élevés, tels que le baccalauréat et les diplômes sanctionnant des études supérieures. Enfin, le ratio résidences principales/résidences occasionnelles est respecté ${ }^{6}$, mais l'écart est réduit dans notre échantillon. Plusieurs catégories de résidents occasionnels ont été atteintes, notamment les touristes, les excursionnistes et les travailleurs saisonniers (tabl. 2).

À la manière de l'INSEE et pour éviter d'avoir des erreurs d'analyse à cause d'un trop faible effectif ces différentes catégories ont été regroupées en une seule. Dans notre étude nous considérons donc les expressions résidents occasionnels et résidents secondaires comme synonymes. Les différences entre résidents principaux et occasionnels sont minimes en termes d'âge, de statut d'activité et de secteur d'activité. L'on remarquera cependant des diplômes plus élevés chez les résidents secondaires. De plus, les analyses statistiques bivariées et multivariées montrent que le caractère résident occasionnel est souvent proche de celui de fréquentation quotidienne de la mer dans le cadre des loisirs. Ce qui est moins le cas pour les résidents permanents.

Nous présenterons ci-dessous les principaux résultats issus de ces données. En un premier temps, nous aborderons les principaux indicateurs

\begin{tabular}{|c|c|c|c|c|c|}
\hline $\begin{array}{l}\text { Statut de } \\
\text { résidence }\end{array}$ & Effectif & $\%$ & $\%$ & Effectif & $\begin{array}{l}\text { Statut de } \\
\text { résidence }\end{array}$ \\
\hline Principal & 218 & 48,1 & 48,1 & 218 & Principal \\
\hline Secondaire & 198 & 43,7 & 51,9 & 235 & \multirow{4}{*}{$\begin{array}{l}\text { Secondaire } \\
\text { ou } \\
\text { Occasionnel }\end{array}$} \\
\hline Touriste & 25 & 5,5 & 51,9 & 235 & \\
\hline Excursionniste & 10 & 2,2 & 51,9 & 235 & \\
\hline Saisonnier & 2 & 0,4 & 51,9 & 235 & \\
\hline Ensemble & 453 & 100 & 100 & 453 & Ensemble \\
\hline
\end{tabular}

Tableau 2 : Types de résidents interrogés Types of surveyed residents

6. En 2013, l'INSEE comptabilise $35 \%$ de résidences principales, contre $61 \%$ logements occasionnels (total : 31489 logements.) Dans notre enquête, nous avons atteint 421 logements, dont $49 \%$ principaux et $51 \%$ occasionnels. 
de culture du risque, en un deuxième temps, nous évoquerons les éléments décrivant le découplage entre les habitants et leur milieu naturel et enfin nous exposerons les principaux facteurs susceptibles de faire varier cette culture du risque.

\section{Éléments de culture du risque}

Conformément à l'hypothèse stipulant que « le risque est une construction sociale », d'après les résultats de notre enquête, les attitudes des individus face aux aléas répondent à des logiques qui ne relèvent pas directement du milieu. Par exemple, nous avons eu l'occasion d'observer des évaluations du risque opposées chez deux résidents voisins : l'un se sentant exposé et l'autre pas. Dans les trois sites cartographiés ci-dessous (fig. 4 - planche $\mathbf{V}$ ), on peut repérer des points qui représentent les résidences enquêtées. Dans certains cas, des points voisins (entourés en orange) ont des couleurs différentes : les résidents ont deux perceptions opposées des risques de submersion, malgré leurs caractéristiques environnementales identiques, toutes choses étant égales par ailleurs. Dans ce cas, la localisation des individus par rapport aux aléas ne semble pas a priori influencer de façon déterminante leurs attitudes. Ce fait a été constaté par plusieurs autres auteurs : C. Heitz (2005 et 2009), H. Rey-Valette et al. (2012), A.-P. Hellequin et al. (2013) font explicitement état de cette caractéristique.

Au final, notre étude n'a pu mettre en évidence aucun facteur influençant les évaluations du risque. Cependant, nous avons relevé que certaines variables contextuelles contribuent statistiquement à la variation de trois autres variables décrivant la culture du risque : la connaissance des aléas côtiers, les stratégies de gestion préférées des aléas côtiers ainsi que les changements d'attitude suite à des événements extrêmes. Avant d'évoquer dans quelle mesure les réponses à ces questions varient, nous présenterons d'abord les résultats de chacune des questions associées à ces thèmes à l'échelle de l'échantillon.

\section{Connaissances limitées des documents officiels et des événements extrêmes}

Au cours de notre enquête, nous avons évalué la connaissance des causes des aléas par rapport aux informations délivrées par les institutions publiques, notamment celles contenues dans les Documents d'Information Communaux sur les Risques Majeurs (DICRIM). La formulation exacte de la question était : «selon vous, quelles sont les causes des inondations par la mer $^{7}$ ? » Les réponses à cette question ont nécessité une phase de dénombrement assez longue afin de les rendre statistiquement exploitables. En effet, 411 modalités différentes ont été recueillies. De ce fait, nous avons procédé progressivement à des regroupements pour aboutir à deux modalités principales : les causes naturelles (les plus citées : $67 \%$ ) et les causes humaines. Si l'on ramène ces causes à l'échelle des individus (en sachant qu'un individu pouvait citer plusieurs causes), l'on se rend compte que les individus citent souvent soit 1 ou 2 causes, ce qui est relativement plus faible, par rapport à celles citées dans les documents d'information adressés aux habitants. Dans la version courante du DICRIM (MEDDE, 2013), on répertorie 5 causes : on parle de vents violents, de basse pression, de fort coefficient de marée, de vagues et de dépassement de barrières naturelles ou d'ouvrages. Cette situation semble en accord avec le fait que seulement $30 \%$ des individus interrogés ont lu au moins un document officiel (plans de prévention et de sauvegarde confondus) au sujet des risques côtiers, malgré le fait que les 8 communes oléronaises diffusent régulièrement ce genre d’information. Cela nous amène à penser que la portée de cette documentation paraît plutôt faible. Au-delà de cet aspect, les individus paraissent avoir une vision simpliste de la nature de ces phénomènes, citant des causes tranchées : soit naturelles, soit humaines, mais rarement les deux.

En ce qui concerne la connaissance des Plans de Prévention des Risques, on remarquera souvent des décalages entre discours officiels et discours des résidents. La figure 6 (planche VI) est une compilation de réponses à la question : « jusqu'où la mer pourrait-elle se propager en cas d'inondation? "Plus une zone coloriée en bleu est foncée, et plus elle a été citée comme étant submersible. Dans le quartier de la Côtinière, dans la commune de Saint-Pierre-d'Oléron, sur la côte ouest, l'on constatera que les zones les plus citées sont celles

\footnotetext{
7. La formulation « inondation par la mer » a été choisie suite à une phase préliminaire de test au cours de laquelle les personnes interrogées nous demandaient souvent de préciser l'expression «submersion marine».
} 


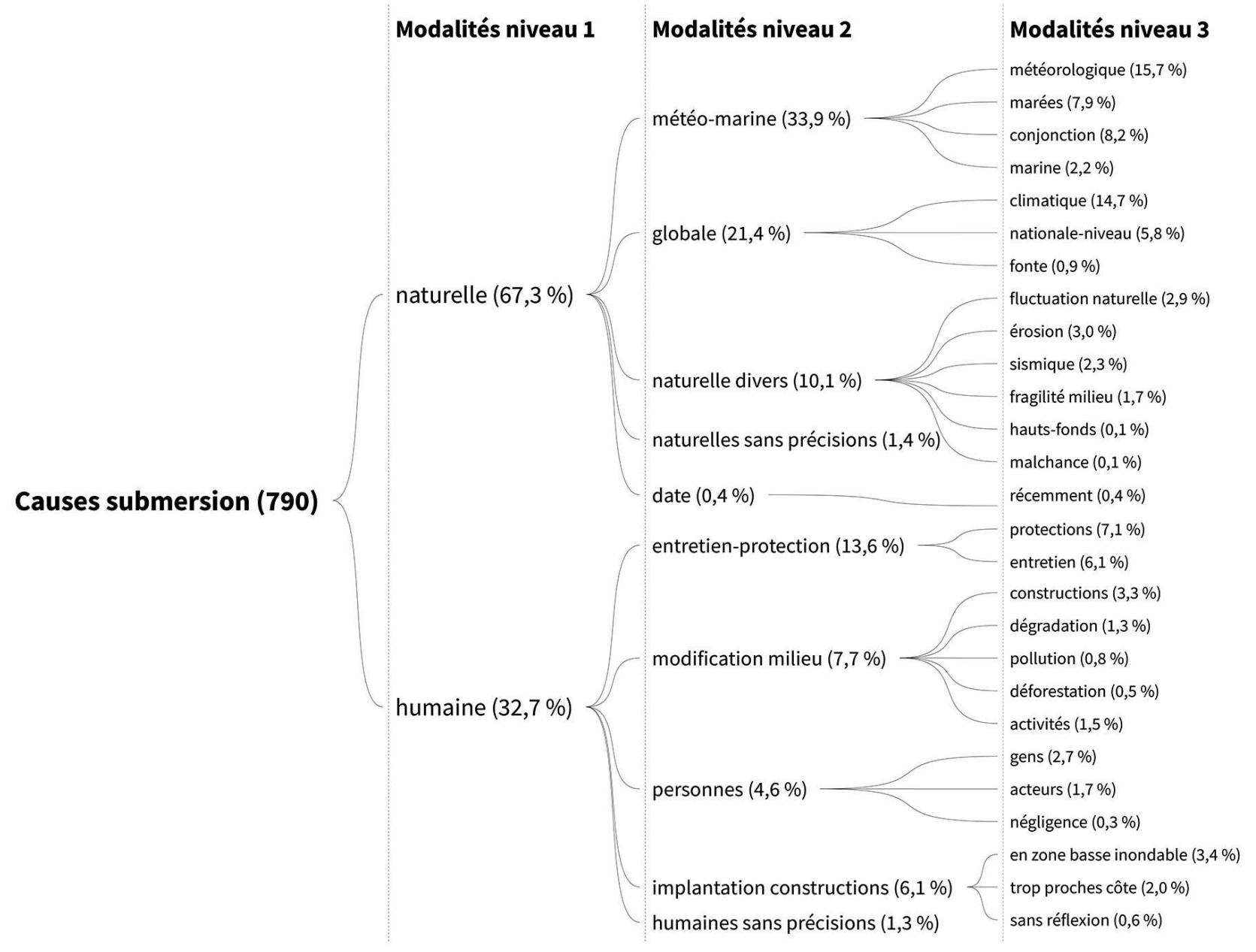

Figure 5 : Classement des causes des aléas de submersion marine

Classification of the given-causes concerning marine flooding hazards

qui sont les plus proches de la mer. Les personnes interrogées semblent ne pas tenir compte des zones basses (anciennement des marais) au sud du quartier, indiquées sur le PPR. Par ailleurs, cette carte montre aussi que la plupart des individus estiment les risques de submersion davantage en fonction de la distance à la mer que par rapport à la topographie du site. Dans ce cas précis, les habitations les plus proches de la mer sont généralement les plus hautes (altitude supérieure à $6 \mathrm{~m} \mathrm{NGF}$ ), car elles se situent sur d'anciennes dunes alors qu'en s'éloignant du trait de côte, l'altitude décline.

Si les connaissances théoriques nous paraissent décalées, voire faibles, nous avons pu observer aussi une mémoire des événements extrêmes très sélective. Les réponses à la question à propos de la mémoire des événements extrêmes ${ }^{8}$ ayant affecté la côte oléronaise montrent que les événements retenus par les individus sont peu nombreux. Ainsi, comme Anselme et al. (2008) nous observons que les événements les plus cités sont les plus récents. Plus précisément, ceux les plus cités sont les tempêtes de 1999 (37\% des citations) et celle de 2010 $(50 \% \text { des citations })^{9}$. Enfin, au regard des autres événements extrêmes (submersions, érosion, tsunami, séismes, ruptures d'ouvrages), nous avons observé une prépondérance d'événements de type

8. «Pouvez-vous me citer des événements extrêmes dont vous avez eu connaissance ou que vous avez vécus? " Nous n'avons pas précisé ce que nous entendions par « extrême » afin que l'on puisse définir a posteriori ce qui est entendu comme extrême par les individus.

9. Au total, nous avons obtenu 719 citations de 16 événements extrêmes différents, par 453 individus interrogés. 
« tempête ». En effet, 93,2 \% des individus interrogés citent des tempêtes (quelle que soit leur intensité), seulement 13,5\% des submersions marines et $9,5 \%$ de l'érosion.

\section{Comportements : des réactions faibles et inadaptées}

Plusieurs travaux ont montré que des actions entreprises vis-à-vis des aléas restent marginales. Avoir vécu un événement extrême conduit peu les individus à réagir ou à prendre n'importe quelle disposition afin d'améliorer leur sécurité. (Heitz, 2005, 2009; RINAMED, 2004; Hellequin et al., 2013; Meur-Férec et al., 2011). Seulement $22 \%$ des personnes interrogées ont déclaré avoir changé leur façon de vivre. Si l'on s'intéresse plus précisément aux changements, $1 / 3$ des déclarations peuvent se considérer comme étant des changements individuels concrets, les autres étant plus des changements d'état d'esprit, voire de recherche d'informations. À l'image des conclusions du programme RINAMED (2004), nous constatons que les individus semblent éprouver des difficultés à dépasser le stade du changement de conscience et de recherche d'informations.

Confrontés à une situation de crise, les individus montrent des réactions particulières. En ce qui concerne les choix de routes d'évacuation lors d'une éventuelle submersion, la figure 7 (planche VII) montre que les individus choisissant de quitter leur logement se réfugient soit vers le centre de l'île, soit sur le continent (Philippenko, 2016; Marlet, 2016). Si cela peut paraître raisonnable, la plupart des personnes empruntent en réalité des routes potentiellement, voire déjà, submergées lors de la tempête Xynthia. Par exemple, la majorité des individus souhaitent quitter l'île en passant par le pont, empruntant la route départementale susceptible d'être submergée au niveau d'Ors. De même, les individus quittant Boyardville préfèrent se réfugier à Saint-Pierre en passant par les marais, alors que le point haut le plus proche se trouve quelques centaines de mètres au nord, dans le massif dunaire des Saumonards. Ces choix suggèrent une méconnaissance du milieu.

\section{Un découplage habitant-milieu confirmé}

Comme nous l'avions annoncé en introduction, une hypothèse forte de cette étude proposait qu'une partie importante de la population oléronaise, du fait de son renouvellement, présente une relation particulière au milieu et serait, par conséquent, susceptible de détenir des connaissances et des comportements inadaptés. D'après notre enquête, 3 questions décrivent cette relation. Il s'agit de la manière dont les individus décrivent la mer, leurs préoccupations quotidiennes ainsi que leurs stratégies préférées de gestion des risques côtiers. Dans cette partie, nous commencerons par présenter les réponses à ces questions; ensuite, nous vérifierons si celles-ci influent sur les discours des individus.

À la question «si l'on vous dit mer, quels sont les trois premiers mots qui vous viennent à l'esprit », le corpus de mots obtenu laisse apparaître une conception globalement positive et stéréotypée de la mer (fig. 8 - planche VIII). Plus des $3 / 4$ des individus associent à la « mer » des termes positifs. Plus précisément, les mots les plus cités font référence à des pratiques et à des traits caractéristiques de l'île d'Oléron. Ainsi, le terme le plus récurrent (76 occurrences) est «pêche». Ce terme désigne les pratiques de pêche à la ligne et à pied. Ensuite, nous avons «plage » (59), «vacances » (54), «baignade» (53). Le premier terme négatif est « danger » et il n'est cité que 18 fois. Il n'y a que 3 autres termes négatifs : «tempête », «peur» et «érosion ». Ainsi, seulement 1 individu sur 10 proposera à la fois des mots connotés positivement et négativement (c'està-dire qu'il parlera par exemple de danger, érosion, inondation, peur et tempête, même si les tempêtes peuvent, parfois, être considérées comme belles, et donc connotées positivement). Cette tendance se retrouve dans des études similaires, telles que celles conduites au sein du programme CRISSIS (Anselme et al., 2016), ou par Rey-Valette et al. (2013).

Concernant la place des risques côtiers au quotidien, nous avons pu observer que l'on nous a parlé principalement de "réseau routier saturé ", de " surpopulation l'été (tourisme) » et du « mauvais état des routes ». Les risques côtiers ne représentent que $14,3 \%$ des préoccupations citées. Les individus ont principalement des préoccupations sociétales, que l'on pourrait retrouver dans d'autres milieux urbains. 
Par ailleurs, en ce qui concerne les stratégies préférées de lutte contre les aléas côtiers, trois catégories principales ont été citées. Le maintien du trait de côte reste la stratégie préférée (cité par $73 \%)$, qu'elle implique des méthodes d'ingénierie lourde ou souple. L'adaptation des enjeux est citée par $12 \%$ : elle concerne notamment une implantation raisonnée du nouveau bâti. Enfin, le recul, impliquant la destruction et la relocalisation des enjeux existants exposés, n'est cité que par $3 \%$. Cette préférence pour la fixation du trait de côte et la protection des enjeux existants a été constatée par plusieurs auteurs tels que S. Deffossez (2006), A.-P. Hellequin et al. (2013), C. Meur-Férec (2011) et N. Krien (2014).

Ainsi, ces réponses montrent une représentation de la mer plutôt optimiste, des préoccupations majoritairement sociales et une volonté de maîtriser les aléas plus qu'à limiter ou adapter les enjeux, allant à l'encontre de la nature fluctuante du littoral. Un découplage entre les résidents et leur milieu semble donc confirmé. Néanmoins, les tests d'indépendance n'ont pas montré de lien entre cette relation particulière au milieu et les composantes de culture du risque.

\section{Des facteurs spatiaux peu influents}

Cependant, les Analyses en Composantes Multiples nous ont permis de mettre en évidence trois types de variables contextuelles influençant la culture du risque : les variables sociodémographiques; les sources d'information et les pratiques de loisirs.

Les variables de nature sociodémographiques telles que l'âge et les diplômes induisent les variations de la CDR les plus significatives. Ainsi les catégories de populations les moins âgées (entre 40 et 60 ans) et les plus diplômées ont tendance à fournir les réponses les plus riches (par exemple en citant des causes de submersion mixtes), sont plus favorables à des stratégies d'adaptation du trait de côte et sont généralement plus réactives suite à des événements extrêmes. On notera que l'âge et les diplômes sont fortement liés : les catégories les moins âgées sont les plus diplômées et inversement.

En ce qui concerne les sources d'information, les individus s'informant à travers l'administration publique et disposant de sources variées (notam- ment émanant de spécialistes ou de proches) sont ceux qui exprimeront les réponses les plus substantielles. Dans le cas où les individus disposent de sources limitées et qu'ils suivent essentiellement des médias généralistes, leurs discours seront plus superficiels. Les ACM, ont montré par ailleurs que les individus citant l'administration en tant que source auront tendance à citer aussi des sources spécialisées, mettant en évidence un groupe d'individus particulièrement bien informés. Par ailleurs, on remarquera aussi que le fait d'avoir vécu un événement extrême ne semble par jouer un rôle dans la qualité des réponses. Cela met indirectement en exergue le rôle important de la communication.

Enfin, certaines variables décrivant des liens particuliers à l'espace semblent avoir un impact sur les discours. En particulier, les résidents occasionnels et fréquentant quotidiennement le bord de mer, dans le cadre de loisirs, délivreront les réponses les plus riches et seront plus réactifs face aux aléas côtiers. De plus, les individus pratiquant des loisirs dont l'environnement est l'objet (par exemple des activités d'observation de la nature, de chasse, pêche ou cueillette) seront aussi plus réactifs face aux aléas côtiers. Enfin, les résidents secondaires, pratiquant plus souvent les loisirs cités précédemment, paraissent être mieux préparés aux risques côtiers que les résidents principaux. Ces résultats remettent en cause l'idée que les individus ayant vécu plus longtemps dans un endroit donné disposeraient des meilleures connaissances de celui-ci. Cela est aussi confirmé par le fait que les corrélations entre la durée de fréquentation du littoral et leur culture du risque sont peu significatives. Par ailleurs, le fait que les résidents secondaires détiendraient de meilleures connaissances se retrouve dans les études récentes sur cette catégorie de la population (Blondy et al., 2016).

\section{Discussion ET CONCLUSION}

Les résultats obtenus à partir de ce travail doivent tenir compte de certaines limites. En particulier, la taille relativement faible de l'échantillon nous a posé régulièrement problème en termes d'analyses. Cela nous a obligé à simplifier les discours recueillis. Ensuite, même si leur proportion est conforme à la population oléronaise de référence, les individus âgés de moins de 40 ans sont très peu représentés. 
Enfin, si le regroupement des résidents secondaires et des vacanciers ne semble pas avoir perturbé les résultats des analyses, il aurait été plus correct de les séparer.

L'ensemble des résultats permet cependant d'affirmer que le point de vue des populations locales est sensiblement différent de celui de l'État en ce qui concerne les connaissances et les pratiques. Celles-ci ne pourront s'accorder qu'au prix d'un changement important de leur manière de voir et d'agir face aux aléas côtiers. En particulier leur rapport à l'environnement que nous avons pu décrire à travers des représentations, mais aussi des pratiques. Nous pensons qu'il est difficile de chercher à vouloir inculquer une nouvelle manière de voir les choses, dans ce cas une meilleure conscience des risques, alors que les pratiques quotidiennes des individus paraissent éloignées de la nature, de l'idée d'une nature complexe faite non seulement de beau, mais aussi d'écueils.

Au cœur de l'idée de «culture du risque » selon l'État apparaît l'objectif d'informer les populations au sujet des risques qu'ils encourent afin qu'ils développent une conscience et des comportements adaptés. D’après les éléments recueillis pour ce travail, actuellement la quantité d'informations disponible à ce sujet est notable, cependant l'intérêt pour ces informations reste faible. Nous pensons que cela est dû au fait que leur acceptation nécessiterait de remettre en question les relations que les individus entretiennent avec la Nature et les institutions. Il s'agit alors d'amener les individus à repenser leur conception des risques afin d'aller audelà du caractère " peu enrôlant » des directives institutionnelles (Goutx, 2012) et de concilier les conflits existants entre les individus et l'État, du fait de leurs conceptions différentes de la problématique des risques littoraux. Plusieurs chercheurs ont d'ailleurs travaillé sur ces rapports conflictuels : Anckière et Langumier (2009); Tricot, Labussière (2008); Weiss et al. (2011).

Dans ce cadre, le concept de « culture » peut apporter des pistes d'amélioration à ces problèmes. Cela permettrait aussi à l'expression de « culture du risque » de mieux incarner les éléments et les dynamiques qu'elle est censée identifier. L'exploration de la définition de "culture » n'est pas aisée du fait qu'il en existe une multitude en sciences humaines (Kroeber et al., 1952). Néanmoins, cet ouvrage de synthèse montre qu'il existe deux groupes de théories sur l'origine de la culture : le premier considère la culture comme une invention créée pour résoudre des questions liées à la survie et le second estime que la culture est une libre invention, qui se place au-delà de toute contrainte biologique. Dans le cadre de notre analyse, nous pensons que le premier groupe est le mieux adapté. Ainsi, nous pouvons repérer certains aspects récurrents dans les définitions de culture :

- une culture désigne ce qui est de l'ordre de l'acquis, à la différence de ce qui est inné;

- elle a pour objet la survie d'un groupe donné. Elle comprend ainsi des éléments :

pour assurer le maintien d'un ordre donné parmi les individus du groupe;

pour assurer la survie par rapport à un environnement donné;

- ces acquis sont des connaissances basées sur des expériences ou des croyances déterminant les « meilleures » manières de voir et d'agir par rapport à un environnement donné (au quotidien ou dans des situations particulières);

- ces connaissances sont transmises et entretenues d'une génération à l'autre par des échanges qui vont du simple dialogue à des rites plus ou moins complexes.

Il est important de noter que la culture est efficace par rapport à un environnement de référence. Celui-ci est constitué à la fois d'éléments naturels (ressources, dangers) et sociaux (amis, ennemis). La modification d'un ou plusieurs éléments de l'environnement de référence peut rendre une culture inadaptée ${ }^{10}$. Ces modifications peuvent survenir dépendamment ou indépendamment du groupe donné. L'on peut penser par exemple à changements du milieu naturel ou à des invasions. Ou alors des facteurs propres au groupe peuvent en être la cause : des connaissances insuffisantes ou des pratiques qui se révèlent inadaptées, mais aussi la migration du groupe vers un nouvel environnementdifférent dans lequel la culture de départ se montrerait inefficace.

Ces précisions nous permettent de mieux cerner l'état d'une éventuelle culture du risque auprès des populations exposées à des aléas côtiers. En effet, une culture des risques côtiers semble théoriquement exister dans la mesure où :

10. Et par conséquent, elle peut conduire à l'effondrement du groupe concerné (Diamond, 2006). 
- elle est un ensemble de connaissances et de pratiques, censées déterminer une meilleure conscience et de meilleurs comportements par rapport aux aléas côtiers (point 3 de la liste précédente);

- et qu'elle a pour objectif de réduire la vulnérabilité des populations en situation de crise, mais aussi de prévenir ces situations à risque (point 2.b);

- l'efficacité de cette culture permettrait, par ailleurs, de préserver une certaine paix sociale ${ }^{11}$ (point 2.a).

En effet, cet ensemble existe a priori. Cependant, la définition de culture proposée met à nouveau en évidence des insuffisances au niveau de l'acquisition des connaissances par les populations. Compte tenu des déclarations des principales instances gouvernementales, les populations ne semblent pas porter d'intérêt à ces informations et peu paraissent en avoir connaissance. Au-delà de la communication institutionnelle, nous nous interrogeons sur la manière dont les individus se saisissent directement ou indirectement des problématiques liées aux aléas côtiers.

Ainsi, l'adaptation à un environnement donné ne peut atteindre un résultat correct si l'on ne dispose pas de « clés » permettant à un individu de déterminer correctement la nature des éléments qui le composent et, par conséquent, d'agir efficacement. L'acquisition de connaissance d'un environnement donné peut se faire selon deux méthodes :

- une confrontation directe avec l'environnement (expérience);

- une confrontation indirecte via des intermédiaires (mémoire).

Cependant, avec l'éradication de l'impact d'aléas fréquents, mais de faible intensité et la période de relative accalmie des phénomènes naturels lors de la deuxième moitié du $\mathrm{xx}^{\mathrm{e}}$ siècle, évoquée par Pigeon (2010), les événements extrêmes sont certes fortement médiatisés, mais peu d'individus au final ont été directement confrontés à ces phénomènes. Et cela risque de poser problème dans la mesure où l'expérience directe apparaît comme étant la meilleure manière de déterminer le comportement le plus adapté (Goutx, 2012).

La mémoire apparaît comme étant un élément central de la culture du risque. En effet, les prin-

11. Ce point est en théorie valide, mais les réactions des individus illustrent une situation défectueuse. cipaux rapports gouvernementaux insistent sur l'importance de tenir compte des aléas passés au cours de l'élaboration des PPR, si leur emprise est supérieure aux aléas simulés. De plus, ils appellent à augmenter la pose des repères des PHEC suivant la directive européenne 2007/60/EC du 23 octobre 2007. Au-delà de la connaissance des aléas, des auteurs tels que Labeur (2013), Acerra et Sauzeau (2012) montrent l'importance de la mémoire pour le partage et l'entretien des expériences. Ils illustrent aussi que souvent des ruptures sont observées dans la transmission de ces savoirs, ce qui semble être le cas pour les résidents de l'île d'Oléron.

En reprenant les apports du concept de «culture », on se rend compte que la culture du risque selon l'État est inadaptée, non pas à cause d'une modification du milieu naturel, mais parce que des caractères propres aux individus ont changé, comme nous l'avons montré à plusieurs reprises. Ainsi peuvent s'expliquer les échecs récurrents des stratégies de communication et de sensibilisation de l'État français. Celui-ci tend à concevoir le manque de connaissances ou de conscience comme étant un manque d'informations. Comme le montrent A. Lammel et al. (2012), le problème est plus complexe : «l'information n'est pas connaissance». Dans ces conditions, nous pensons qu'il est primordial de continuer à étudier les rapports entre les sociétés et leurs milieux, et en particulier de rendre compte de leurs évolutions.

\section{Remerciements}

Cette recherche a bénéficié du soutien de la Fondation de France.

\section{Bibliographie}

Acerra Martine, Sauzeau Thierry, 2012. Zones construites, zones désertes sur le littoral atlantique. Les leçons du passé, Norois, no 222, p. 103-114.

Anckière Marie, Langumier Julien, 2009. La remise en eau de la plaine de Piolenc-Mornas face à la constitution d'une culture locale de l'arrangement, VertigO, v. 9, n 1

Anselme Brice (coord.), 2016. Caractérisation des Risques Submersion marine sur des Sites Sensibles, rapport final, 145 p.

Anselme Brice, Goeldner-Gianella Lydie, Durand Paul, 2008. Le risque de submersion dans le système lagunaire 
de La Palme (Languedoc, France) : nature de l'aléa et perception du risque, Actes du colloque international pluridisciplinaire Les littoraux : subir, dire et agir, 16-18 janvier 2008, Lille.

AnZiAni Alain (rapporteur), 2010. Xynthia : une culture du risque pour éviter de nouveaux drames, rapport d'information fait au nom de la mission commune d'information sur les conséquences de la tempête Xynthia, nº 647, Sénat, 227 p.

Blondy Caroline, Vacher Luc, Vye Didier, 2016. Les résidents secondaires, des acteurs essentiels des systèmes touristiques littoraux français?, Territoire en mouvement Revue de géographie et aménagement, $\mathrm{n}^{\circ} 30,21 \mathrm{p}$.

Cartier Stéphane, 2004. Implications du public face aux risques naturels : délégation aux pouvoirs publics ou construction locale des politiques participatives?, in DE Larjartre A., Gaboriau V., Les collectivités territoriales face aux risques physiques, Paris, L'Harmattan, p. 141-155.

D'Ercole Robert, 1996. Représentations cartographiques des facteurs de vulnérabilité des populations exposées à une menace volcanique. Application à la région du volcan Cotopaxi (Équateur), Bulletin de l'Institut français des études andines, $\mathrm{n}^{\circ} 25$, v. 3, p. 479-507.

De Vanssay Bernadette, Colbeau Justin L., Marchand D., Vallette C., Bakhache C., Poumadère M., 2011. Construire une mémoire sociale utile de la tempête Xynthia du 28 février 2010, Académie de l'eau, Ministère de l'Écologie, du Développement durable, des Transports et du Logement, $137 \mathrm{p}$.

Deffossez Stéphanie, 2006. Réflexion pour une réduction de la vulnérabilité face aux inondations. L'exemple des basses plaines de l'Aude, in LeOne F., Vinet F., La vulnérabilité des sociétés et des territoires face aux menaces naturelles. Analyses géographiques, Université Paul-Valéry - Montpellier 3, p. 125-131.

Douglas Mary, Wildavsky Aaron, 1982. Risk and culture: an essay on the selection of technical and environmental dangers, University of California Press, 221 p.

Duvat-Magnan Virginie, 2010. Les impacts de la tempête Xynthia sur les plages de l'île d'Oléron: les réalités du terrain, rapport, $29 \mathrm{p}$.

Garnier Emmanuel (rapporteur), Boucard Jacques, Surville Frédéric (coordination), 2010. La crise Xynthia à l'aune de l'histoire. Enseignements et enjeux contemporains d'une histoire des submersions, Contribution aux missions d'enquête parlementaire et sénatoriale sur Xynthia par le groupe de recherche SUBMERSIONS, $112 \mathrm{p}$.

Goutx David, 2012. Rôle des individus dans la prévention des risques d'inondation et la gestion de crise, $23^{\text {es }}$ Journées Scientifiques de l'Environnement - Risques environnementaux : détecter, comprendre, s'adapter, Janvier 2012, Créteil, France, $25 \mathrm{p}$.

Heitz Carine, 2005. Étude de la perception du risque de catastrophes naturelles relatif aux coulées de boue par les acteurs de communes périurbaines. Approche méthodologique et analyse d'enquêtes (Sundgau, Alsace), Mémoire de DEA, Laboratoire «Image, Ville et Environnement ", Université de Strasbourg, $110 \mathrm{p}$.
Heitz Carine, 2009. La perception du risque de coulées boueuses: analyse sociogéographique et apports à l'économie comportementale, Thèse de doctorat, Université de Strasbourg, $299 \mathrm{p}$.

Hellequin Anne-Peggy, Flanquart Hervé, Meur-Férec Catherine, Rulleau Bénédicte, 2013. Perceptions du risque de submersion marine par la population du littoral languedocien : contribution à l'analyse de la vulnérabilité côtière, Natures Sciences Sociétés, n² 1, p. 385-399.

IFEN - Institut Français de L'environnement, 2007. Le littoral, entre nature et artificialisation croissante, Le 4 pages, $\mathrm{n}^{\circ} 120$.

Joffe Hélène, 2003. Risk : From perception to social representation, British Journal of Social Psychology, n. 42, p. 55-73.

Krien Nathalie, 2014. Place des risques côtiers dans la représentation du cadre de vie d'individus possédant des enjeux sur des communes «à risque », Thèse de doctorat, Université de Bretagne Occidentale, $246 \mathrm{p}$.

Kroeber Alfred Louis, Kluckhohn Clyde, Untereiner Wayne, Meyer Alfred G., 1952. Culture, a critical review of concepts and definitions, Cambridge Mass, The Museum, 223 p.

Labeur Christine, 2013. Raconter l'inondation : quand les récits de catastrophes se font mémoire du risque, Géocarrefour, v. 88 , p. 45-54

Lammel Annamaria, Dugas Emilie, Guillen Gutierrez Elisa, 2012. L'apport de la psychologie cognitive à l'étude de l'adaptation aux changements climatiques : la notion de vulnérabilité cognitive, VertigO, vol. 12, nº 1, 22 p.

Le Bourhis Jean-Pierre, 2007. Du savoir cartographique au pouvoir bureaucratique. Les cartes des zones inondables dans la politique des risques (1970-2000), Genèses, v.3, n. 68 , p. $75-96$.

Marlet Victoire, 2016. Synthèse et analyse de cartes mentales sur le risque de submersion marine : le cas de l'âle d'Oléron, Rapport de stage, Université Paris 1 Panthéon-Sorbonne, École Nationale des Sciences Géographiques, 31 p.

MEDDE - Ministère de L'Écologie, du Développement Durable ET DE L'ÉNERGIE, 2013. Information sur les risques majeurs : DDRM et DICRIM. Maquette nationale, 360 p.

Meschinet de Richemond Nancy, Reghezza Magali, 2010. La gestion du risque en France : contre ou avec le territoire?, Annales de géographie, v. 3, nº 673, p. 248-267.

Meur-Férec Catherine, Flanquart Hervé, Hellequin AnnePeggy, Rulleau Bénédicte, 2011, Risk perception, a key component of systemic vulnerability of coastal zones to erosion-submersion. Case study on the French Mediterranean coast, Littoral 2010. Adapting to Global Change at the Coast: Leadership, Innovation, and Investment, $8 \mathrm{p}$.

Morel Valérie, Deboudt Philippe, Herbert Vincent, LonguépéE Jérôme, Meur-Férec Catherine, 2004. L'ambivalence de l'eau, vecteur d'aménités et de risques, sur les territoires côtiers, Acte du séminaire "Les territoire de l'eau », Université d'Artois, 26 mars 2004, Revue électronique Développement Durable et Territoires, p. 142-155.

Moscovici Serge, 1961. La psychanalyse, son image et son public, Paris, PUF, 652 p. 
Péret Jacques, Sauzeau Thierry, 2014, Xynthia, ou la mémoire réveillée. Des villages charentais et vendéens face à l'océan (XVII ${ }^{e}$-XXI $I^{e}$ siècle), La Crèche, Geste, 296 p.

Peretti-Watel Patrick, 2000. La société du risque, Paris, La Découverte, $126 \mathrm{p}$.

Philippenko Xénia, 2016. La perception du risque de submersion marine et sa représentation cartographique: le cas de l'île d'Oléron, Mémoire de Recherche, Université Paris 1 Panthéon-Sorbonne, 152 p.

Pigeon Patrick, 2010. Catastrophes dites naturelles, risques et développement durable : utilisations géographiques de la courbe de Farmer, VertigO, v. 10, no 1, 13 p.

Rey-Valette Hélène, Fraysse Nicolas, Richard Alexandre, Carbonnel Philippe, 2013. Perception des plages et des politiques de rechargement : réflexions à partir du cas du golfe d'Aigues-Mortes (Hérault/Gard), Espace, Populations, Sociétés, no 1, v. 2, p. 177-192.

Rey-Valette Hélène, Rulleau Bénédicte, Meur-Férec Catherine, Flanquart Hervé, Hellequin Anne-Peggy, SourisSEAU Emmanuelle, 2012. Les plages du littoral languedocien face au risque de submersion : définir des politiques de gestion tenant compte de la perception des usagers, Géographie, économie, société, no 4, v. 14, p. 369-392.

RINAMED - RIsques NAturels dans le MÉDiterranéen occidental (Programme INTERREG 33-Ouest), 2004.
Étude sur la perception des risques naturels en Méditerranée, rapport conclusif, $21 \mathrm{p}$.

RINAMED - RIsques NAturels dans le MÉDiterranéen occidental (Programme INTERREG 33-Ouest), 2004. Stratégies d'appréciation de la perception du risque hydrogéologique dans des zones exposées et lignes directives pour la communication des risques naturels, Rapport conclusif, $217 \mathrm{p}$.

Stovic Paul, 2000. The perception of risk, Earthscan, 473 p.

SOeS - Service de l'Observation et des Statistiques, 2011. Indicateur : perspectives d'évolution de la population des départements littoraux à l'horizon 2040, Observatoire National de la Mer et du Littoral, 5 p.

Tricot Anne, Labussière Olivier, 2008. Cultures du risque : de la doctrine officielle aux expériences plurielles des cours d'eau, Géocarrefour, no 81, 12 p.

VINET Freddy, 2007. Approche institutionnelle et contraintes locales de la gestion du risque. Recherches sur le risque inondation en Languedoc-Roussillon, Habilitation à Diriger des Recherches, Université de Montpellier 3 Paul Valery, v. 3, $330 \mathrm{p}$.

Weiss Karine, Girandola F., Colbeau-Justin L., 2011. Les comportements de protection face au risque naturel: de la résistance à l'engagement, Pratiques Psychologiques, v. 17, $n^{\circ} 3$, p. 251-262. 